1. Introduction

Frequency independent behavior of the fundamental antenna parameters such as gain, reflection coefficient and radiation pattern is an important issue and is always considered in the applications requiring very wide bandwidths [1-8]. If the frequency behavior of spiral, bi-conical and log-periodic antennas is examined it is seen that those parameters do not vary much with the frequency and therefore they can generally be classified as frequency independent antennas $[9,10]$. Although they are classified as frequency independent, the independency is limited to a frequency band which is determined by the antenna size [10-12]. Since spiral antennas can be constructed as planar structures and radiate circularly polarized waves, they are widely used in remote sensing (Ground Penetrating Radar etc.), direction finding (Global Positioning Systems etc.), telemetry, and flush mounted airborne applications when compared to the others $[1,5,7]$. A spiral antenna is usually designed by using Archimedean [1-5] or logarithmic [11, 13] geometries. Although Archimedean spiral antennas may have wide operation bandwidths, they cannot be categorized as completely frequency independent according to Rumsey's scaling principle [9]. One can obtain a more frequency independent antenna by using a logarithmic spiral because it satisfies the requirements given in the scaling principle. The disadvantage of using a log-spiral in the antenna designs is that it becomes very large even in the first few turns. Since the antenna is located in a very limited place, the turns become very close to each other and the configuration leads to a very dense structure around its center. Therefore implementing and feeding such an antenna is not very suitable because of the practical considerations.

Spiral antennas are designed and fabricated in round or square geometries. When they are examined one can see that the square spiral antennas have the advantage of operating with the same performances at lower frequencies [1,14]. First radiation band of a spiral antenna occurs when the circumference of the spiral is one wavelength, corresponding to a diameter $D=\lambda / \pi$ for the circular one and a width $W=\lambda / 4$ for the square one. Therefore the first operation frequency is approximately $20 \%$ lower for a square spiral, when compared with that of the round one if they are about to be the same size, but square geometries seem to be more frequency dependent at high frequencies [15]. Another important point which must be considered in the designs is the arm length of spiral antenna. If the small number of turns and short arm lengths are employed the antennas are found to be more frequency dependent $[9,10]$. In such cases the end of the arms are terminated with loads to prevent the reflections causing to a decrease in bandwidth and deterioration in axial ratio $[1,4$, 16]. Since spiral antennas are generally printed on dielectric 14 substrates for the physical support and they can be loaded by dielectric materials to improve their radiation characteristics $[17,18]$, the interaction of the spiral antenna with the dielectric substrate is also an important issue and the effect of dielectric on the radiation characteristics must also be investigated.

In this study, it is intended to design square spiral antennas, which are not dense around the feed point, operating in a broadband with small number of turns and without using any terminations at their ends. The proposed geometries are first employed to wire and strip antennas in free-space and then a dielectric substrate is added to the strip antenna to examine the effect of physical support on the radiation characteristics. All of the antennas obtained from the proposed geometries are also compared with the Archimedean one in terms of the fundamental antenna parameters. Experimental study has also been performed by using the slot antenna for the comparisons and verification of the numerical results.

\section{Antenna design}

The equation defining the well known geometry of a round Archimedean spiral used in antenna designs is given as

$\rho=a \alpha+b$

where $\rho$ and $\alpha$ are the conventional polar coordinates in the $x-z$ plane ( $\rho$ is the distance mesured from the origin and $\alpha$ is the angle measured from the $x$ axis), $a$ is the growth rate and $b$ is the starting point of spiral curve on the $x$ axis. From the equation it is obvious that the Archimedean spiral arms have a constant amount of growth rate at each turn. Square spiral approximates the round Archimedean spiral in this manner and therefore it always has the same distance between its arms as can be seen in Figure 1.

Unlike the Archimedean spiral, logarithmic spiral given as

$\rho=b e^{a \alpha}$

satisfies the requirements for the Rumsey's scaling principle. As a result of that the round logarithmic spiral can be approximated to a square spiral, as in the Archimedean case, to obtain a more frequency independent square spiral antenna. Since the logarithmic spiral arms have an exponential growth rate, the antenna becomes very large even in the first few turns and therefore the resulting structure is not a good candidate for the implementation due to the reasons mentioned in the previous section. For an antenna which is easier to implement, the log-spiral can be approximated in a different manner such that it also preserves its frequency independent behavior. The new spiral can be obtained by increasing its arm distance from the feed point only at the 


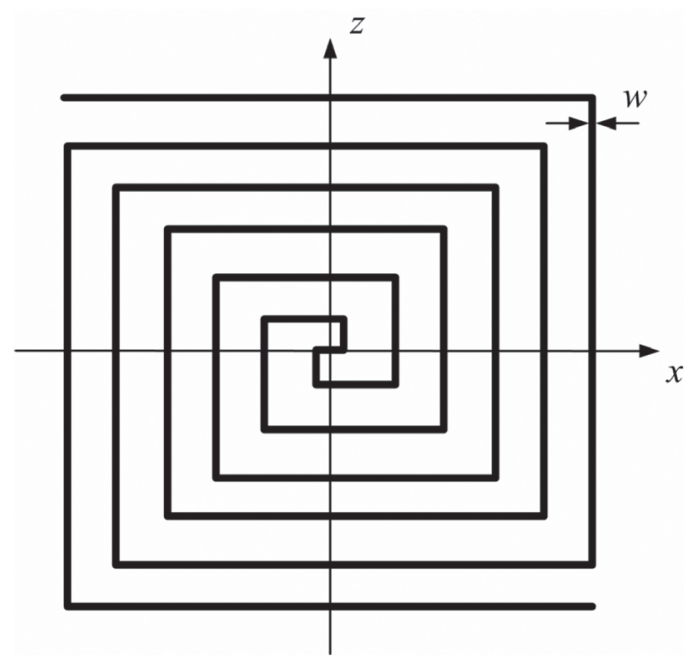

Fig. 1: Square Archimedean spiral.

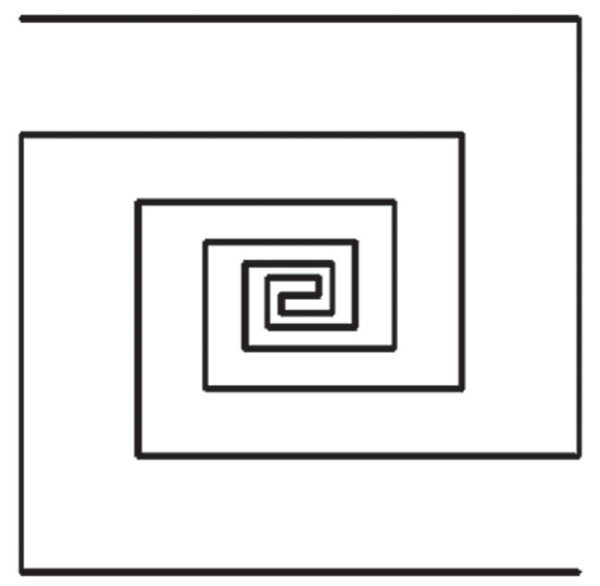

Fig. 2: Modified square logarithmic spiral.

second and fourth quadrant. The resulting configuration can be called as the modified square logarithmic spiral and it is shown in Figure 2.

If the configuration is examined it can be seen that the length and location of each straight segment in one direction are determined according to a scale factor $k$ which is given as

$k=\frac{l_{n+1}}{l_{n}}=\frac{R_{n+1}}{R_{n}}$

where $l_{n}$ is the length of the $n^{\text {th }}$ segment and $R_{n}$ is its distance from the reference point. By this approach the geometry obtained is not very dense around its center as in the exact approximation of the log-spiral, and since it again has the logarithmic properties one can also conclude that the antenna designed by using this geometry is expected to be more frequency independent.

To improve the radiation characteristics, another configuration for the square spiral antenna can be obtained by a special arrangement of its arms. The first arm of spiral expands as in the modified square logarithmic one and the second arm is arranged to be placed exactly between the two consecutive turns of the first arm. Even though the second arm expands with the scale factor $k$ like the first arm and preserves the logarithmic properties, the whole spiral becomes very different when compared to the modified square log-spiral. Consequently, because of this special arrangement, it is neither an approximation of the round Archimedean spiral nor an approximation of the round logarithmic spiral. Since some of

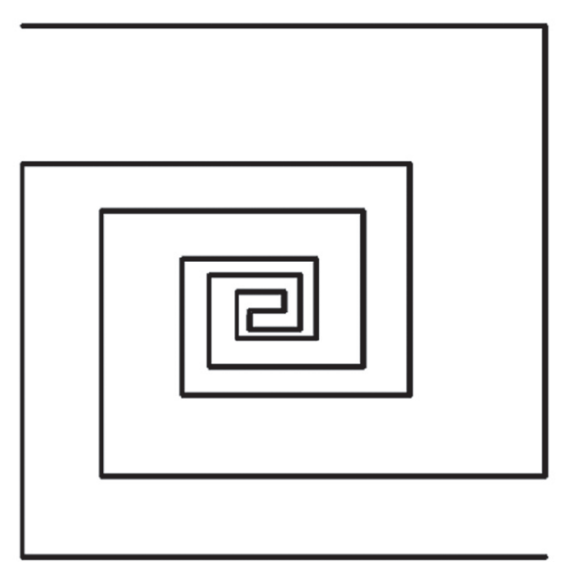

Fig. 3: Modified square Archimedean-logarithmic hybrid spiral.

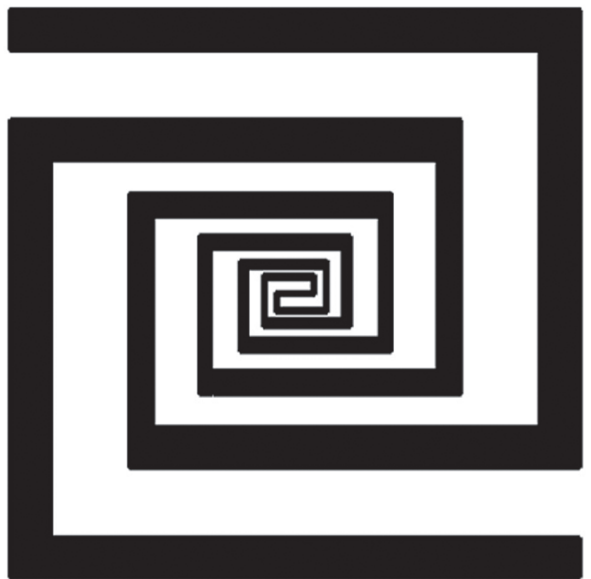

Fig. 4: Modified square logarithmic spiral with variable segment widths.

its parts are similar to the Archimedean spiral geometry and some parts are similar to the logarithmic spiral geometry, the proposed geometry can be interpreted as an approximation of both and can be called as Modified square Archimedeanlogarithmic hybrid spiral which is illustrated in Figure 3. .

To obtain another antenna having all of the logarithmic properties, the width of the $n^{\text {th }}$ straight segment, $w_{n}$, can also be chosen as

$k=\frac{w_{n+1}}{w_{n}}$

in accordance with (3). The resulting geometry, obtained by using the modified square log-spiral and (4), is shown in Figure 4. .

The geometry seen in the figure can be thought as the square approximation of the self complementary round logarithmic spiral but if carefully examined it is seen that it is actually a very different configuration because of the new design method employed.

\section{Numerical and experimental results}

In this section, fundamental parameters such as gain, axial ratio, directivity and voltage standing wave ratio (VSWR) are examined for wire, strip and slot antennas. In the numerical analysis results obtained for the proposed designs are compared with those of the Archimedean one. To make a complete comparison between the performances of the antennas, design parameters such as the number of turns for 

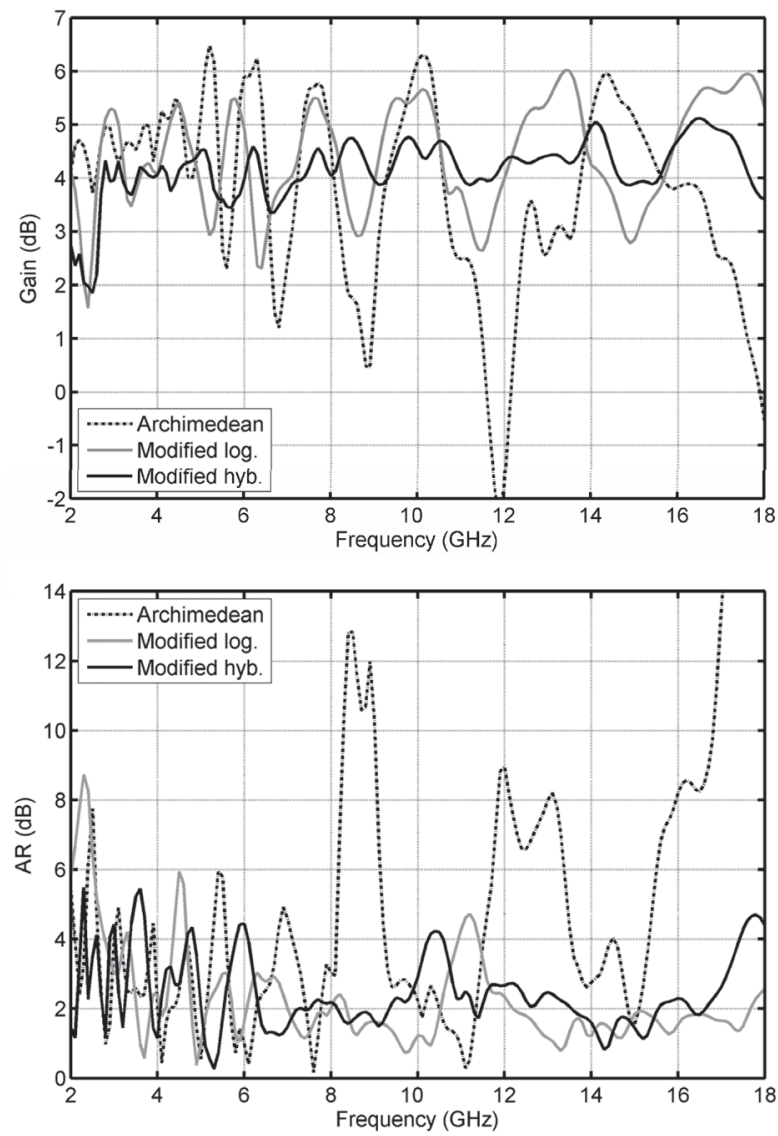

Fig. 5: Gains and axial ratios for the Archimedean, Modified logarithmic and Modified hybrid spiral wire antennas.

the spirals, the area covered by the antennas and their feed points are chosen as to be exactly the same for all cases. The results for wire and strip antennas are obtained in a frequency band from $2 \mathrm{GHz}$ to $18 \mathrm{GHz}$ by using the Method of Moments (MoM) and Finite Difference Time Domain Method (FDTD), respectively $[19,20]$. The spirals used in both cases have $2+7 / 8$ turns and the feed gap for the antennas is always equal to $4.6 \mathrm{~mm}$ at their centers. All of the antennas are located in the $x-z$ plane and they have an area of 8 $\mathrm{cm} \times 8 \mathrm{~cm}$. The radius is $0.1 \mathrm{~mm}$ and each segment length is taken as $\lambda / 10$ at the upper simulation frequency for the wire structures. The cell dimensions used in the analysis of strip structures are chosen to be equal to the strip width as $\Delta x=\Delta y=\Delta z=0.4 \mathrm{~mm}$ for free space simulations. The scale factor $k$ is equal to 1.7 for the proposed antennas. When the dielectric substrate, its thickness is equal to $1.6 \mathrm{~mm}$ and its dielectric constant $\varepsilon_{r}$ is 2.2 , is used to support the antennas, the cell dimension $\Delta y$ in the direction of its thickness is decreased to $0.2 \mathrm{~mm}$. The time step values are also chosen according to the cell sizes.

The antenna gains and axial ratios for the wire antennas are presented in Figure 5. On-axis polarization in forward direction is plotted as a function of frequency in the figure. As can be seen, the proposed antennas are less frequency dependent than the Archimedean one. Extreme variations in the gain and axial ratio of the Archimedean spiral antenna are not present in those of the proposed antennas. The directivities [10] of the antennas at $5 \mathrm{GHz}, 10 \mathrm{GHz}$ and $15 \mathrm{GHz}$ are shown in Figure 6 and they are plotted in the $\varphi= \pm 90^{\circ}$ planes for $0^{\circ}<\theta<180^{\circ}$.

From the figures, one can conclude that both of the proposed antennas perform much better than the conventional Archimedean antenna in terms of the frequency independency of

16 the characteristic parameters but the performance of the

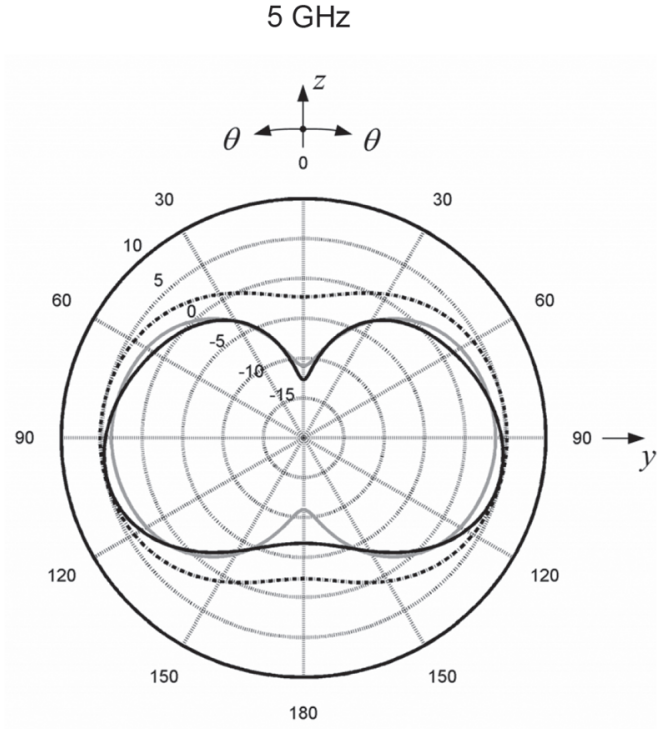

$10 \mathrm{GHz}$

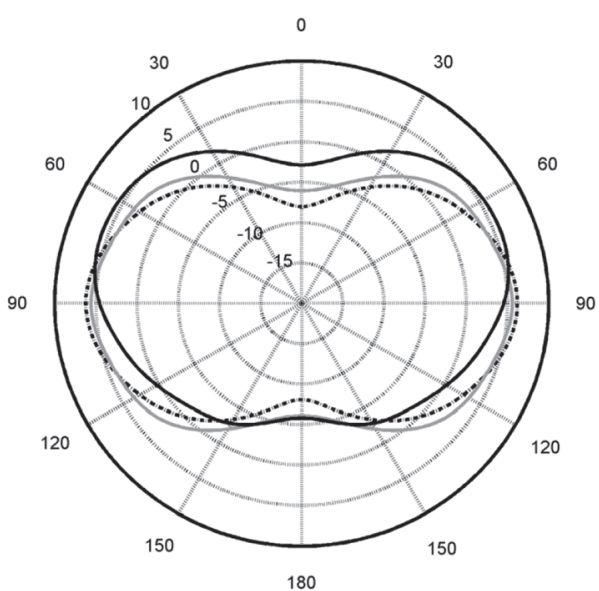

$15 \mathrm{GHz}$

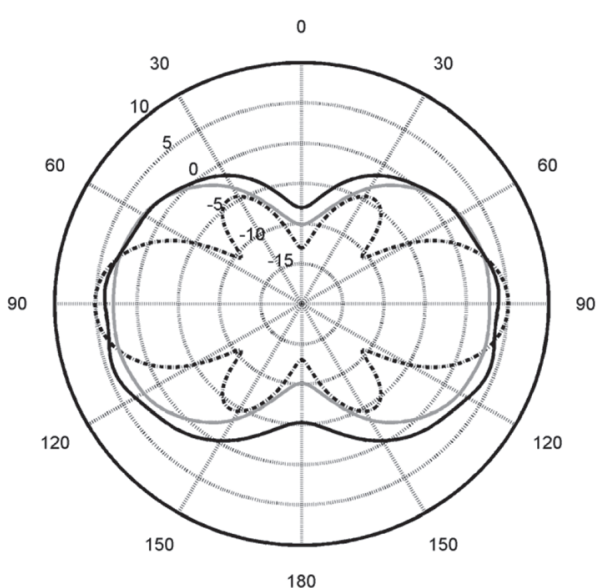

Fig. 6: Directivities for the Archimedean, Modified logarithmic and Modified hybrid spiral wire antennas $\left(\varphi= \pm 90^{\circ}, 0^{\circ}<\theta<180^{\circ}\right.$. Archimedean, Modified log, Modified hyb.).

modified hybrid antenna is the best when compared to the others and therefore it can be a good candidate for the spiral strip antenna applications. The gains and axial ratios of the modified hybrid and Archimedean strip antennas in free space are presented in Figure 7. It is again seen that the proposed antenna performs much better than the other one. 

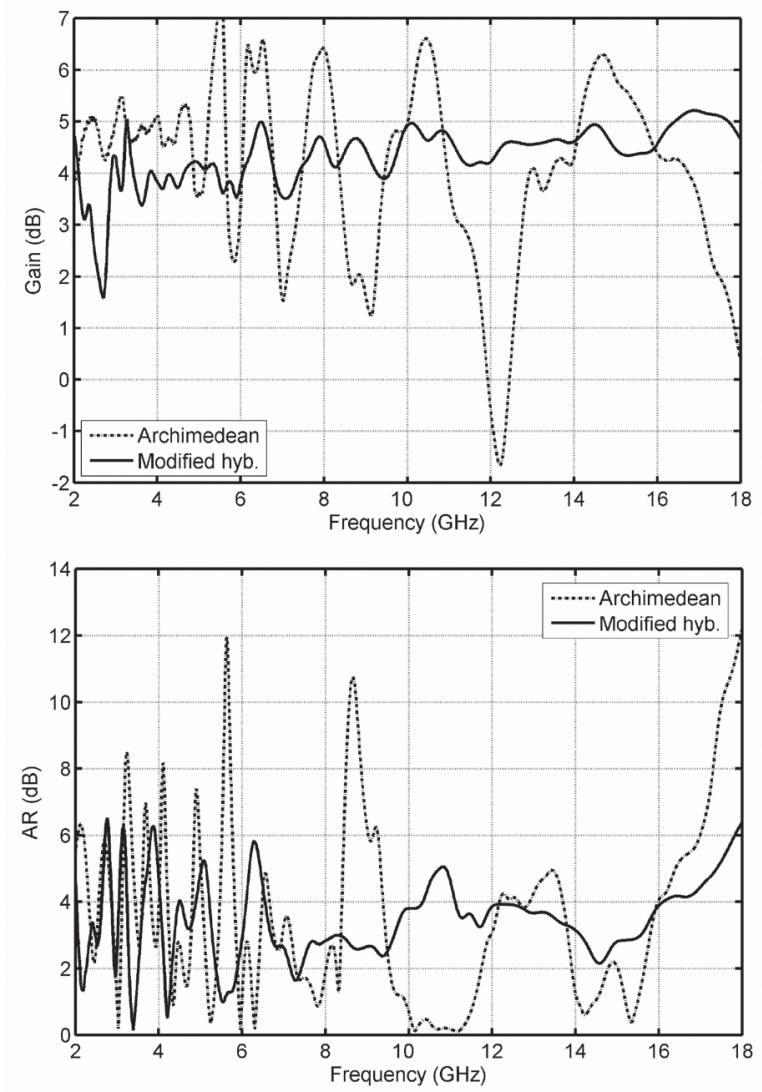

Fig. 7: Gains and axial ratios for the Archimedean and Modified hybrid spiral strip antennas in free space.

Since the strip antennas are generally used with a physical support, a dielectric substrate is added in the next step to support the two antennas examined in the previous case. The results are presented in Figure 8. The effects of substrate on the antenna gains and axial ratios can also be seen by comparing Figure 7 and Figure 8.

From the figures it can be concluded that the gain of Archimedean antenna is much more affected than the proposed one and it decreases drastically at higher frequencies. Another conclusion that can be drawn from the figures is that the dielectric support causes a contraction in both of the gain and axial ratio values in the frequency band. The directivities of antennas at $5 \mathrm{GHz}, 10 \mathrm{GHz}$ and $15 \mathrm{GHz}$, with and without the dielectric support, are presented in Figure 9. If they are examined it can be seen that proposed antenna is less sensitive to frequency. In addition, the directivity of proposed antenna for both cases are similar to each other unlike the Archimedean spiral antenna. These results show that the proposed spiral antenna again has better radiation characteristics when compared to Archimedean one.

The last antenna geometry to be examined is shown in Figure 4 and it is obtained by increasing the widths of the segments. In order to verify the numerical results, experimental study was performed for this structure and the slot version of the antenna is used to achieve a symmetrical and balanced feeding over the broad frequency range. The antenna was fabricated by using a single sided copper clad FR-4 sheet having an area of $30 \mathrm{~cm} \times 20 \mathrm{~cm}$ with $1 \mathrm{~mm}$ dielectric substrate thickness. For ease of fabrication, the slot antenna is chosen as to have $1+7 / 8$ turns with $k=2.2$. The variable width of the slot at its center is equal to $0.7 \mathrm{~mm}$ and it increases according to the scale factor $k$. The dielectric material of FR-4 substrate is also taken into consideration and is included in the FDTD computations in a similar manner as

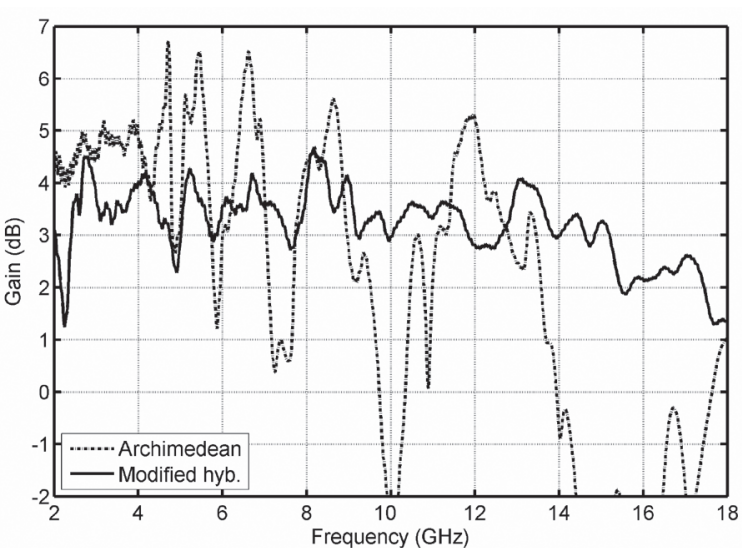

Frequenz 63 (2009) $1-2$

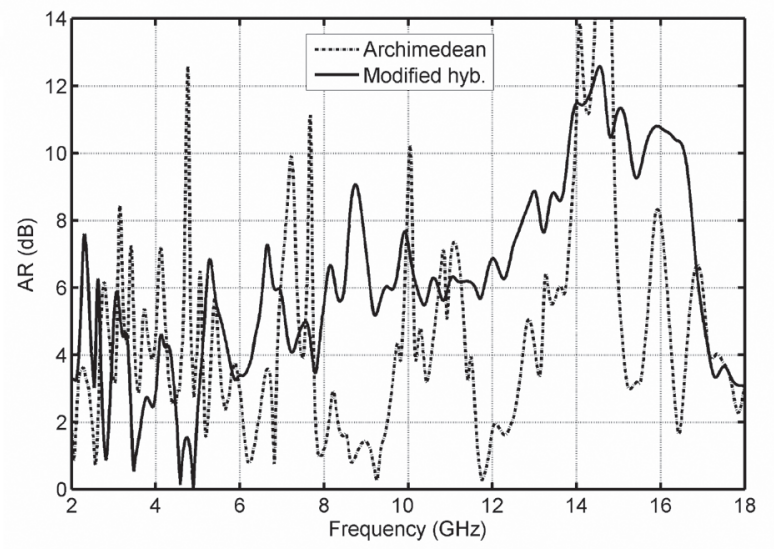

Fig. 8: Gains and axial ratios for the Archimedean and Modified hybrid spiral strip antennas on dielectric substrate.

done for the strip case. The measured and computed values of VSWR for the slot antenna are presented in Figure 10. In the experimental study, the antenna was fed without using a balun and therefore it was not possible to feed the slot antenna structure in a completely balanced manner which causes the discrepancy between the results particularly at lower frequencies. If the results are examined one sees that VSWR is less than 2.5 over a broad frequency band. Especially when the measured values are considered, it is also seen that the 8:1 VSWR bandwidth is achieved for the fabricated antenna and the results are also in good agreement in most of that region as shown in the figure. Although a very small number of turns are used for the spiral, the antenna obtained with this special geometry is not very frequency dependent over the band.

\section{Conclusion}

Novel designs for broadband square spiral antennas have been introduced in this paper. The spiral geometries have been obtained by very special arrangements of their arms. The new and conventional designs have been analyzed and compared by using the MoM and FDTD for the wire, strip and slot antenna structures. The performance of the proposed antennas is better in terms of the frequency independency of characteristic antenna parameters and it is shown that they are very effective especially if the small number of spiral turns is considered. The results obtained from the experimental study have also been compared with the computed ones for the verification of the numerical data. 
Frequenz 63 (2009)

90
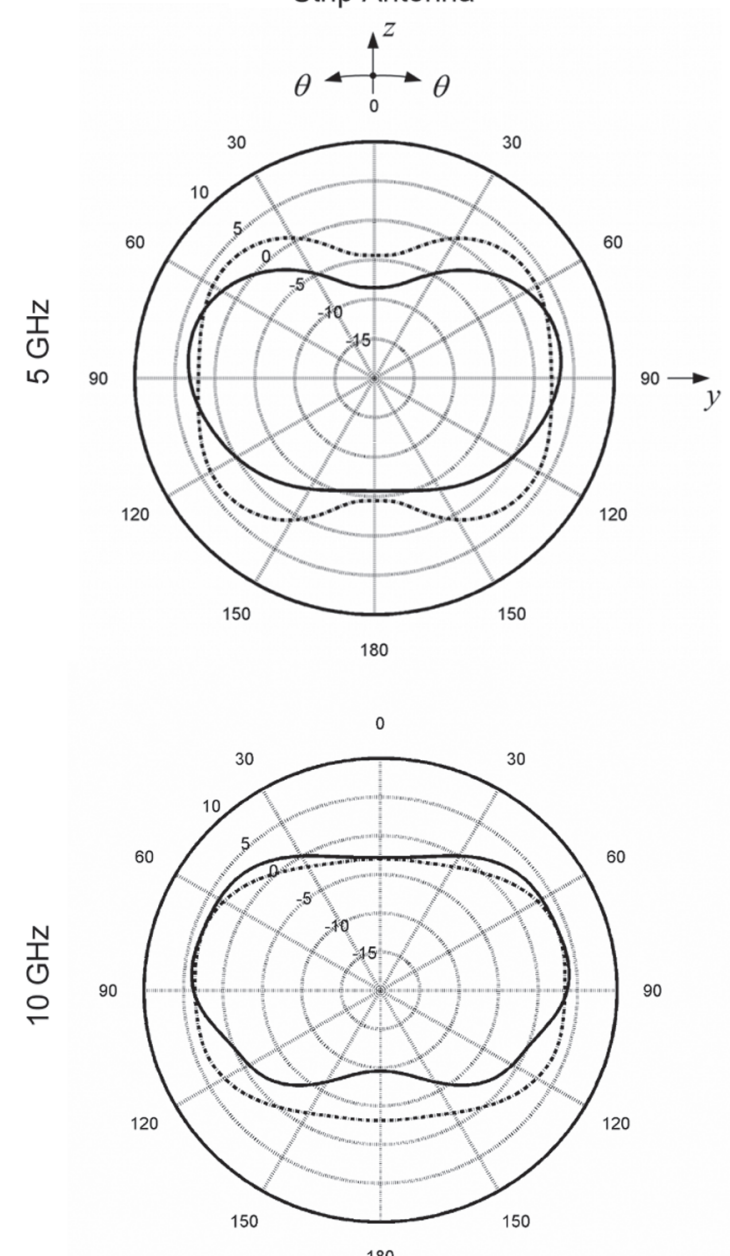

180

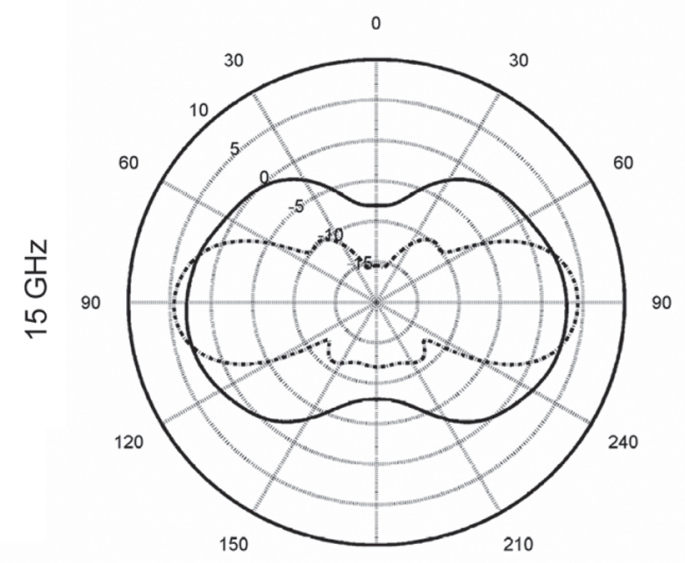

180
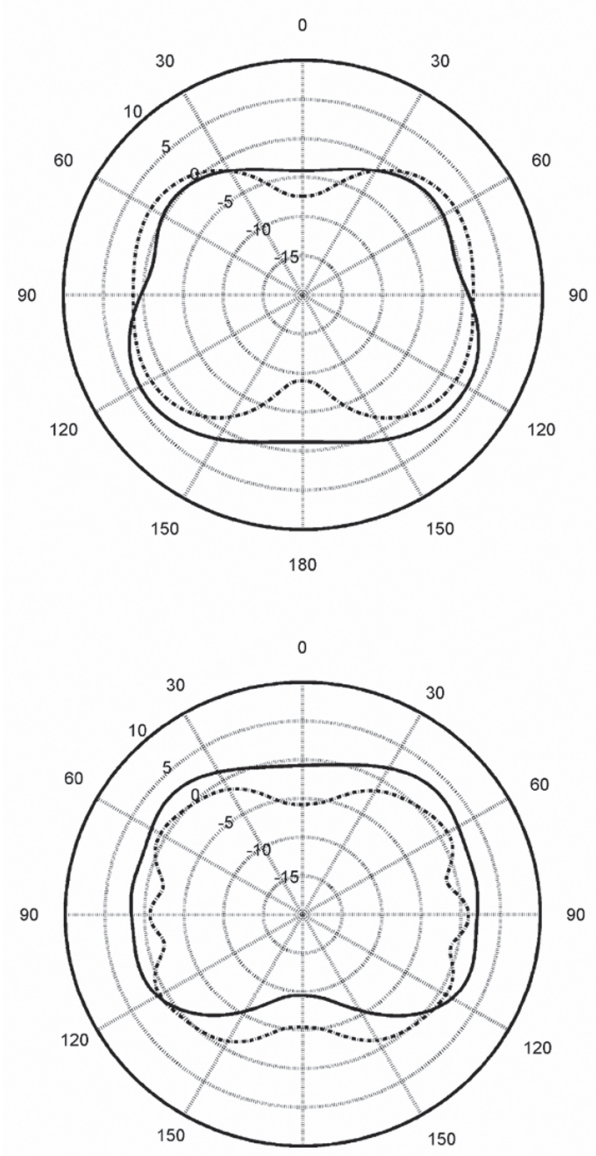

180

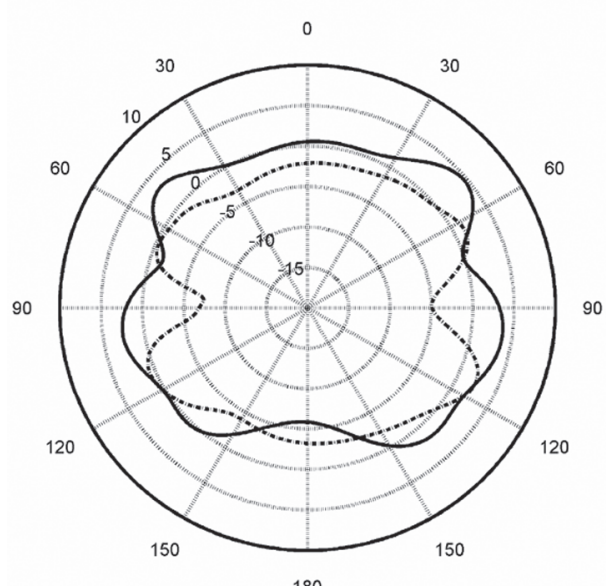

180

Fig. 9: Directivities for the Archimedean and Modified hybrid spiral strip antennas in free space and on dielectric substrate $\left(\varphi= \pm 90^{\circ}, 0^{\circ}<\theta<180^{\circ}\right.$, Archimedean, Modified hyb.).

\section{References}

[1] B.A.Kramer, M. Lee, C.C. Chen and J.L. Volakis, "Design and Performance of an Ultrawide-Band Ceramic-Loaded Slot Spiral," IEEE Trans. on Antennas and Propagat., Vol. 53, No. 7, pp. 21932199, July 2005.

[2] D.S.Filipovic, and J.L.Volakis, "Broadband Meanderline Slot Spiral Antenna," IEE Proc-Microw. Antennas Propagat., Vol. 149, No. 2 , pp. 98-105, April 2002

[3] L. Sevgi and G. Cakir, "A Broadband Array of Archimedean Spiral Antennas for Wireless Applications," Microwave and Optical Tech. Lett., Vol. 48, No. 1, pp. 195-200, January 2006.

[4] M.W. Nurnberger and J.L. Volakis, "Extremely broadband slot spiral antenna with shallow reflecting cavities," Electromagnetics, Vol. 20, No. 4, pp. 357-376, July 2000.
[5] T.A. Iwasaki, P. Freundorfer and K. Iizuka, "A unidirectional semicircular spiral antenna for subsurface radars," IEEE Trans. on Electromagnetic Comp., Vol. 36, No. 1, pp. 1-6, February 1994.

[6] E. R. Brown, A. W. M. Lee, B.S. Navi and J.E. Bjarnason, "Characterization of Planar Self-Complementary Square-Spiral Antenna in the THz Region," Microwave and Optical Tech. Lett., Vol. 48, No. 3, pp. 524-528, March 2006.

[7] J. Ely, C. Christodoulou and D. Shively, "Square Spiral Antennas for Wireless Applications," Proc. IEEE NTC Conference, Orlando, May 1995, pp. 229-232.

[8] J.M. Bell and M.F. Iskander, "A low-profile Archimedean spiral antenna using a EBG ground plane," IEEE Antennas Wireless Propagat. Lett., Vol. 3, No. 1, pp. 223-226, 2004.

[9] V. H. Rumsey, Frequency Independent Antennas, Academic Press, New York and London, 1966. 


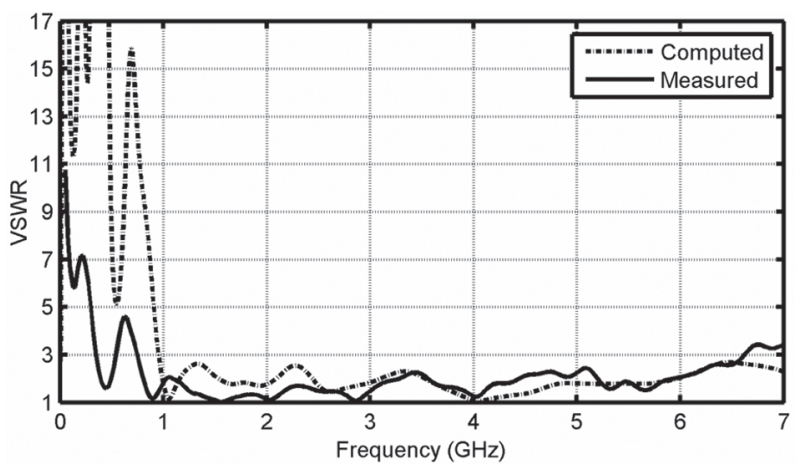

Fig. 10: VSWR of the Modified square logarithmic spiral slot antenna with variable segment widths.

[10] C.A. Balanis, Antenna Theory: Analysis and Design, $2^{\text {nd }}$ Edition, John Wiley and Sons, New York, 1997.

[11] J.D. Dyson, "The Equiangular Spiral Antenna," IRE Trans. on Antennas and Propagat., Vol. 7, No. 2, pp. 181-188, April 1959.

[12] R. T. Gloutak and N. G. Alexopoulos, "Two-Arm Eccentric Spiral Antenna," IEEE Trans. on Antennas and Propagat., Vol. 45, No. 4 pp. 723-730, April 1997.

[13] J. J. H. Wang and V. K. Tripp, "Design of Multioctave Spiral-Mode Microstrip Antennas," IEEE Trans. on Antennas and Propagat., Vol. 39, No. 3, pp. 332-335, March 1991.

[14] R. Bawer, and J.J. Wolfe, "The Spiral Antenna," IRE Int. Convention Record, New York, March 1960, pp. 84-95.

[15] E. D. Caswell, Design and Analysis of Star Spiral with Application to Wideband Array with Variable Element Sizes, Dissertation, Virginia Polytech. Ins., USA, 2001.
[16] M.W. Nurnberger and J.L. Volakis, "New termination for ultrawideband slot spirals," IEEE Trans. on Antennas and Propagat., Vol. 50 No. 1, pp. 82-85, Jan. 2002.

[17] H. Nakano, H. Yasui and J. Yamauchi, "Numerical analysis of twoarm spiral antennas printed on a finite-size dielectric substrate," IEEE Trans. on Antennas and Propagat. Vol. 50, No. 3, pp. 362-370, March 2002.

[18] H. Nakano, M. Ikeda, K. Hitosugi and J. Yamauchi, "A spiral antenna sandwiched by dielectric layers," IEEE Trans. on Antennas and Propagat. Vol. 52, No. 6, pp. 1417-1423, June 2004.

[19] Supernec 2.7 Academic Version, Poynting Software Ltd., www. poynting.co.za.

[20] XFDTD Bio-pro V6.2, Remcom Inc., www.remcom.com.

First Author

Ugur Saynak

Information Technologies Institute

TUBITAK Marmara Research Center

Gebze-41470. Kocaeli, Turkey.

E-mail: ugur.saynak@bte.mam.gov.tr

Second Author

Alp Kustepeli

Dept. of Electrical and Electronics Engineering

Izmir Institute of Technology

Urla-35430. Izmir, Turkey.

E-mail: alpkustepeli@iyte.edu.tr

* This work was supported in part by EU-FP6 INCO "IYTE Wireless" Project, Contract No: 017442
Frequenz $63(2009)$

1-2 